Journal for ImmunoTherapy of Cancer

\title{
Activated osteoarthritis following immune checkpoint inhibitor treatment: an observational study
}

\author{
Pankti Reid (D) , ${ }^{1}$ David FL Liew (D) , ${ }^{2,3}$ Rajshi Akruwala, ${ }^{4}$ Anne R Bass, ${ }^{5}$ \\ Karmela K Chan ${ }^{5}$
}

To cite: Reid P, Liew DFL, Akruwala R, et al. Activated osteoarthritis following immune checkpoint inhibitor treatment: an observational study. Journal for ImmunoTherapy of Cancer 2021;9:e003260. doi:10.1136/ jitc-2021-003260

- Additional supplemental material is published online only. To view, please visit the journal online (http://dx.doi.org/10. 1136/jitc-2021-003260).

Accepted 17 August 2021

Check for updates

(C) Author(s) (or their employer(s)) 2021. Re-use permitted under CC BY-NC. No commercial re-use. See rights and permissions. Published by BMJ.

${ }^{1}$ Medicine, University of Chicago Biological Sciences Division,

Chicago, Illinois, USA

${ }^{2}$ Department of Medicine, The University of Melbourne, Melbourne, Victoria, Australia ${ }^{3}$ Division of Rheumatology and Department of Clinical Pharmacology and Therapeutics, Austin Health, Heidelberg, Victoria, Australia

${ }^{4}$ Department of Medicine, SUNY Downstate Medical Center, New York City, New York, USA

${ }^{5}$ Division of Rheumatology, Hospital for Special Surgery, New York, New York, USA

Correspondence to

Dr Pankti Reid;

pankti.reid@uchospitals.edu

\section{ABSTRACT}

Immune checkpoint inhibitors (ICls) have revolutionized cancer therapy but can result in toxicities, known as immune-related adverse events (irAEs), due to a hyperactivated immune system. ICl-related inflammatory arthritis has been described in literature, but herewith we introduce and characterize post-ICl-activated osteoarthritis (ICl-aOA). We conducted a multicenter, retrospective, observational study of patients with cancer treated with ICls and diagnosed with ICl-aOA by a rheumatologist. ICIaOA was defined by (1) an increase in non-inflammatory joint pain after ICI initiation, (2) in joints characteristically affected by osteoarthritis, and (3) lack of inflammation on exam. Cases were graded using the Common Terminology Criteria for Adverse Events (CTCAE) V.6.0 rubric for arthralgia. Response Evaluation Criteria in Solid Tumors V.1.1 (v.4.03) guidelines determined tumor response. Results were analyzed using $\chi^{2}$ tests of association and multivariate logistic regression. Thirty-six patients had $\mathrm{ICl}-\mathrm{aOA}$ with a mean age at time of rheumatology presentation of 66 years (51-81 years). Most patients had metastatic melanoma (10/36, 28\%) and had received a PD-1/PD-L1 inhibitor monotherapy (31/36, 86\%) with $5 / 36(14 \%)$ combination therapy. Large joint involvement (hip/knee) was noted in $53 \%$ (19/36), small joints of hand $25 \%(9 / 36)$, and spine $14 \%(5 / 36)$. Two-thirds $(24 / 36)$ suffered multiple joint involvement. Three of $36(8 \%)$ had CTCAE grade 3, $14(39 \%)$ grade 2 and $19(53 \%)$ grade 1 manifestations. Symptom onset ranged from 6 days to 33.8 months with a median of 5.2 months after ICl initiation; five patients suffered from $\mathrm{ICl}-\mathrm{aOA}$ after $\mathrm{ICl}$ cessation (0.6, 3.5, 4.4 , 7.3, and 15.4 months after ICI cessation). The most common form of therapy was intra-articular corticosteroid injections only (15/36, 42\%) followed by nonsteroidal anti-inflammatory drugs only $(7 / 36,20 \%)$. Twenty patients (56\%) experienced other irAEs, with rheumatic and dermatological being the most common. All three patients with high-grade $\mathrm{ICl}-\mathrm{aOA}$ also had another irAE diagnosis at some point after ICl initiation. ICl-aOA should be recognized as an adverse event of $\mathrm{ICl}$ immunotherapy. Early referral to a rheumatologist can facilitate the distinction between $\mathrm{ICl}$ induced inflammatory arthritis from post-ICI mechanical arthropathy, the latter of which can be managed with local therapy that will not compromise ICl efficacy.

\section{INTRODUCTION}

Immune-related adverse events (irAEs) constitute emerging autoimmune disease entities that result from off-target toxicity after immune checkpoint inhibitor (ICI) therapy. irAEs span all organ systems and vary in time and duration of presentation as well as severity. ${ }^{1}$ Musculoskeletal toxicities of ICIs range from arthralgias and myalgias to inflammatory AEs with phenotypes akin to many rheumatic diseases such as sicca syndrome, inflammatory myositis, or inflammatory arthritis, as seen with rheumatoid arthritis, spondyloarthritis, and polymyalgia rheumatica. ${ }^{2}$ ICI-inflammatory arthritis (ICI-IA) is the most commonly described rheumatic irAE and can present even after ICI cessation. ${ }^{34}$ Systematic reviews report an incidence of arthralgias after ICI as high as $43 \%$ but of inflammatory arthritis only up to $7 \% .{ }^{256} \mathrm{In}$ recent years, there has been growing literature dedicated to immune-related inflammatory arthritis, but there is still a paucity of research investigating non-inflammatory joint pain that can occur after ICI therapy. ${ }^{3-9}$

To understand the pathomechanisms behind ICI-associated arthralgia, it is vital to differentiate inflammatory presentations of arthritis from those of non-inflammatory or mechanical arthropathy like osteoarthritis $(\mathrm{OA})$. Clinically, the two present with different symptomology and physical exam findings; in addition, management and prognosis differ. Inflammatory arthritis, in its truest form, presents with pain in association with erythema, swelling, and increased warmth with stiffness that predominates during morning hours. ${ }^{10}$ Conversely, symptoms of OA include pain that worsens through the day with increased activity. OA tends to impact weight-bearing or overused joints such as the spine, hips, knees, and the first carpometacarpal joint (CMC) at the base of the thumbs. Despite its noninflammatory clinical presentation, OA has surfaced to be mechanistically complex. ${ }^{11-13}$ Studies implicate low-grade inflammation driven by innate immune system activation, 
which checkpoint inhibitor blockade could theoretically exacerbate. $^{14}$

Through this observational study, we investigate the phenomenon of symptomatic worsening of OA following checkpoint inhibitor therapy, termed ICI-activated osteoarthritis (ICI-aOA), from a clinical vantage point. This is the largest case series of OA enhancement after ICI start.

\section{METHODS}

\section{Patient selection}

This is a retrospective case series of patients with histologically confirmed malignancy treated with an ICI and subsequently diagnosed with an exacerbation of OA. Patients were identified on referral for ICI toxicity evaluation, and all of the patients in this cohort were assessed and treated by a board-certified rheumatologist at one of three independent medical centers: Hospital for Special Surgery in New York, University of Chicago in Chicago, Illinois, and Austin Health in Melbourne, Victoria, Australia. Patients were given ICIs as part of a clinical trial or standard of care, with or without chemotherapy. The time frame for reviewed cases ranged from the approval date of respective patient registries (May 2018, July 2018, and May 2018) until February 2021. Thorough chart review and pertinent data extraction was conducted by a representative rheumatologist from each of the three institutions (KKC, PR, and DL). Patients were excluded if the diagnosis of arthropathy was deemed by the treating rheumatologist to be a type of inflammatory arthritis or of an alternate etiology than OA.

\section{Activated osteoarthritis (aOA) definition and diagnosis}

The diagnosis of post-ICI-aOA was made by a boardcertified rheumatologist. A patient was considered to have ICI-aOA if there was a notable clinical worsening of or onset of new joint pain since the start of ICI and all of the following clinical criteria were met:

1. Symptomatically worse with activity, improved with rest and lacking significant morning stiffness $(\leq 30 \mathrm{~min})$.

2. Involvement of a joint or joints characteristically affected by OA, including but not limited to the following: first CMCs, distal interphalangeal joints (DIPs), and/ or proximal interphalangeal joints (PIPs), knees, hips, cervical, and/or lumbar spine.

3. Absence of physical exam findings for inflammation such as swelling, redness, or warmth.

Of note, patients did not have to have a documented diagnosis of OA preceding ICI therapy.

\section{Treatment and adverse event analysis}

Tumor radiological response was evaluated by Response Evaluation Criteria in Solid Tumors V.1.1 (v.4.03) guidelines at 3 months post-ICI start. irAEs were graded according to the Common Terminology Criteria for Adverse Events (CTCAE) V6.0 grading. High-grade ICI-aOA was defined as CTCAE grade $\geq 3$. Labs and imaging were ordered per discretion of the treating rheumatologist and were not mandatory for inclusion in our study. Particular autoantibodies of interest included antinuclear antibody (ANA) (values equal to or above a titer of 1:80 were considered positive), rheumatoid factor (RF) (values equal to or above $14 \mathrm{IU} / \mathrm{mL}$ were considered positive) and anticyclic citrullinated peptide (CCP) (positive values were determined by individual institutional laboratories). When imaging was available, radiographical findings of $\mathrm{OA}$ involving the clinically symptomatic joint(s) were determined by a board-certified radiologist from the respective institution.

\section{Statistical analysis}

Study data were evaluated and summarized using descriptive statistics with Stata V.15.1. Continuous variables were reported with means and ranges, while categorical and ordinal variables were summarized as percentages. $\chi^{2}$ test was used for categorial data and test of association. Multivariate likelihood ratios with $95 \%$ CIs were computed using a logistic regression model. The threshold for statistical significance was set at a two-sided $\mathrm{p}$ value of $<0.05$.

\section{RESULTS}

\section{Study population and clinical characteristics}

We identified a total of 36 patients with ICI-aOA as summarized in table 1 (detailed in online supplemental table 1). The mean age at ICI-aOA diagnosis was 66 years (range 51-81 years) with 53\% male. Most patients were Caucasian $(34 / 36,94 \%)$, and metastatic melanoma was the most common malignancy $(10 / 36,28 \%)$. While there were no patients treated with CTLA4 inhibitor (CTLA4i) monotherapy, a majority of patients were managed with PD-1/PD-L1 inhibitor (PD-1/PD-L1i) monotherapy $(31 / 36,86 \%)$ and some with combination therapy $(5 / 36$, $14 \%)$.

\section{ICI-aOA characteristics}

About half of the patients had only large joint involvement $(19 / 36,53 \%)$ with knees and hips impacted, while one-fourth had small joints of the hand (first CMC, DIP and/or PIP) involved and less than $10 \%$ had both large and small joint involvement. Two-thirds were identified to have multiple joint involvement $(24 / 36,67 \%)$. Over half of the patients (19 of $36,52.8 \%$ ) had ICI-aOA grade 1 and about $40 \%$ (14 of 36 ) had ICI-aOA grade 2, while only 3 of $36(8 \%)$ patients had high-grade ICI-aOA. Median time of symptom onset after start of checkpoint therapy was 5.2 months with a wide range from 6 days to 33.8 months. Most cases occurred within the first 2-3 months after start of ICI; however, there was another peak of cases around 10 months after ICI start (figure 1A). While most cases occurred during ICI therapy, 5 of 36 (14\%) patients experienced ICI-aOA after ICI cessation $(0.6,3.5,4.4,7.3$, and 15.4 months after ICI cessation). These cases corresponded to 2.0, 9.6, 19.1, 8.7, and 16.1 months after ICI initiation, respectively (portrayed in figure 1A). All five of these patients also had a diagnosis of one or more irAEs. 
Table $1 \mathrm{ICl}-\mathrm{aOA}($ total $=36)$

\begin{tabular}{|c|c|}
\hline \multicolumn{2}{|l|}{ Gender } \\
\hline Female & $17(47.2 \%)$ \\
\hline Male & $19(52.8 \%)$ \\
\hline \multicolumn{2}{|l|}{ Race/ethnicity } \\
\hline Caucasian & $34(94.4 \%)$ \\
\hline African-American & $1(2.8 \%)$ \\
\hline American-Indian & $1(2.8 \%)$ \\
\hline \multicolumn{2}{|l|}{ Age } \\
\hline Mean age at $\mathrm{ICl}-\mathrm{aOA}$ diagnosis (range) & $66(51-81)$ \\
\hline Mean age at cancer diagnosis (range) & $63(43-79)$ \\
\hline \multicolumn{2}{|l|}{ Cancer type } \\
\hline Melanoma & $10(27.8 \%)$ \\
\hline NSCLC & $5(13.9 \%)$ \\
\hline $\mathrm{RCC}$ & $6(16.7 \%)$ \\
\hline Urothelial cancer & $6(16.7 \%)$ \\
\hline Gynecological malignancy & $3(8.3 \%)$ \\
\hline Head and neck & $2(5.6 \%)$ \\
\hline Others & $4(11.1 \%)$ \\
\hline \multicolumn{2}{|l|}{$\mathrm{ICl}$ type } \\
\hline CTLA-4i & $0(0.0 \%)$ \\
\hline PD-1/PD-L1i & $31(86.1 \%)$ \\
\hline Combination & $5(13.9 \%)$ \\
\hline \multicolumn{2}{|l|}{ Size of joints involved } \\
\hline Large (knees and hips) & $19(52.8 \%)$ \\
\hline Small (first CMCs, DIPs and PIPs) & $9(25.0 \%)$ \\
\hline Spine & $5(13.9 \%)$ \\
\hline Small and large & $3(8.3 \%)$ \\
\hline \multicolumn{2}{|l|}{ Number of joints involved } \\
\hline Single & $7(19.4 \%)$ \\
\hline Multiple & $24(66.7 \%)$ \\
\hline Spine & $5(13.9 \%)$ \\
\hline \multicolumn{2}{|l|}{ Severity of arthritis (CTCAE) } \\
\hline Grade 1 & $19(52.8 \%)$ \\
\hline Grade 2 & $14(38.9 \%)$ \\
\hline Grade 3 & $3(8.3 \%)$ \\
\hline \multicolumn{2}{|l|}{ Presence of other irAEs } \\
\hline Yes & $21(58.3 \%)$ \\
\hline No & $15(39.5 \%)$ \\
\hline
\end{tabular}

Any serological positivity (serology checked in 27 of 36 total patients)

Yes $11(40.7 \%)$

Antinuclear antibody (7/22)

$\mathrm{RF}(3 / 27)$

CCP $(3 / 24)$

RF and/or CCP $(5 / 27)$

No

$16(59.3 \%)$

Cancer outcome

Complete response

$16(44.4 \%)$
Table 1 Continued

\begin{tabular}{lc}
\hline Partial response & $5(13.9 \%)$ \\
\hline Stable disease & $11(30.6 \%)$ \\
\hline Progressive disease & $4(11.1 \%)$ \\
ICl-aOA treatment & \\
\hline Local CSI only & $16(44.4 \%)$ \\
\hline NSAIDs only & $7(20.0 \%)$ \\
\hline Physical therapy only & $7(20.0 \%)$ \\
\hline Systemic corticosteroids & $1(11.4 \%)$ \\
\hline Systemic and local corticosteroids & $3(8.6 \%)$ \\
\hline $\begin{array}{l}\text { Other agents (acetaminophen, duloxetine } \\
\text { and muscle relaxant) }\end{array}$ & $3(8.6 \%)$ \\
Viscosupplementation & $2(5.7 \%)$ \\
\hline Steroid-sparing agent (HCQ) & $1(2.9 \%)$ \\
ICl-aOA outcome & \\
\hline Improved or stabilized & $32(88.9 \%)$ \\
\hline Worsened & $4(11.1 \%)$ \\
\hline Time of symptom onset and clinical follow-up & \\
\hline $\begin{array}{l}\text { Median time of symptom onset after ICI } \\
\text { start }\end{array}$ & 5.2 months (IQR \\
\hline $\begin{array}{l}\text { Median length in rheumatology clinic } \\
\text { follow-up }\end{array}$ & 1.6 months (IQR 0-7, \\
\hline
\end{tabular}

$\mathrm{CCP}$, cyclic citrullinated peptide; $\mathrm{CMC}$, carpometacarpal joint; CSI, corticosteroid injection; CTCAE, Common Terminology Criteria for Adverse Events; CTLA-4i, cytotoxic T lymphocyte-associated protein 4 inhibitor; DIP, distal interphalangeal joint; $\mathrm{HCQ}$, hydroxychloroquine; $\mathrm{ICl}$, immune checkpoint inhibitor; $\mathrm{ICl}-\mathrm{aOA}, \mathrm{ICl}$-activated osteoarthritis; irAE, immune-related adverse event; NSAID, non-steroidal antiinflammatory drug; NSCLC, non-small cell lung carcinoma; PD-1/ $\mathrm{PD}-\mathrm{L} 1 \mathrm{i}$, programmed cell death protein 1 or programmed cell death protein ligand 1 inhibitor; PIP, proximal interphalangeal joint; RCC, renal cell carcinoma; RF, rheumatoid factor.

\section{Laboratory analysis and imaging}

Laboratory evaluation focused on autoantibody testing of ANA, RF and CCP, along with $\mathrm{C}$ reactive protein (CRP) measures. Twenty-seven patients had at least one of the three serologies ordered and of these, $41 \%(11 / 27)$ had positivity. All three patients with high-grade ICI-aOA had at least one of these antibodies positive, and while patients with high-grade ICI-aOA had higher likelihood of serological positivity (Likelihood ratio 5.19, $\mathrm{p}$ value $=0.023$ ), this association did not hold true when adjusted for age, race, and gender. About two-thirds of patients with CRP measured had an elevation $(20 / 31,65 \%)$. Imaging was obtained for 24 patients and demonstrated characteristic changes of those seen in OA. Representative images are displayed in figure 2 and further described in online supplemental table 1.

\section{Presence of other irAEs}

Developments of all irAEs were recorded for our cohort. Twenty patients $(56 \%)$ of a total of 36 patients experienced one or more irAEs at some point after ICI initiation (online supplemental figure 1). Eight patients had more than one other irAE reported. Most commonly noted 
A Timing of Post-ICl Activated Osteoarthritis (ICl-aOA)

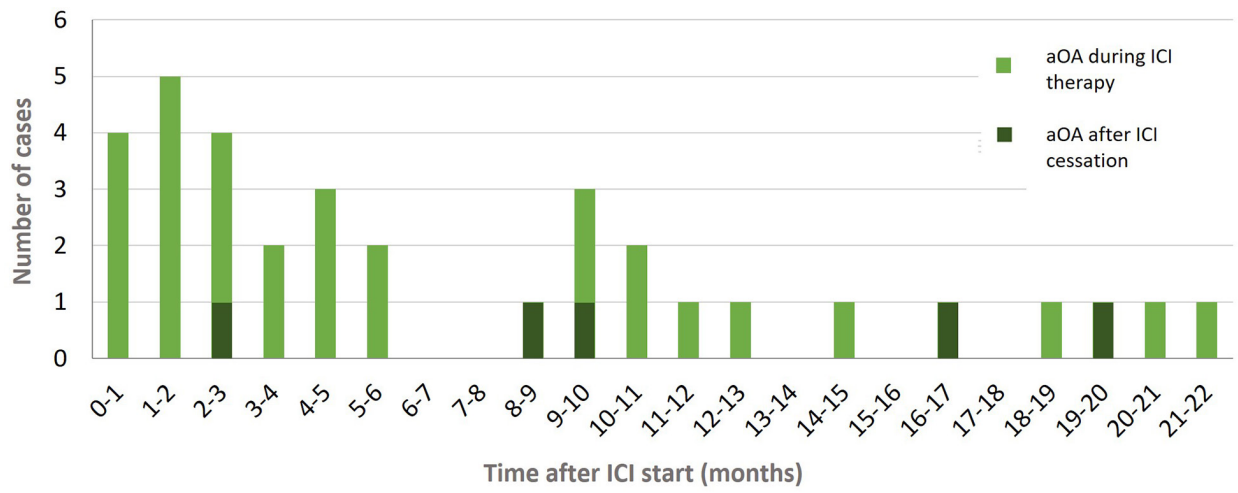

B Therapies for Post-ICl Activated Osteoarthritis (ICl-aOA)

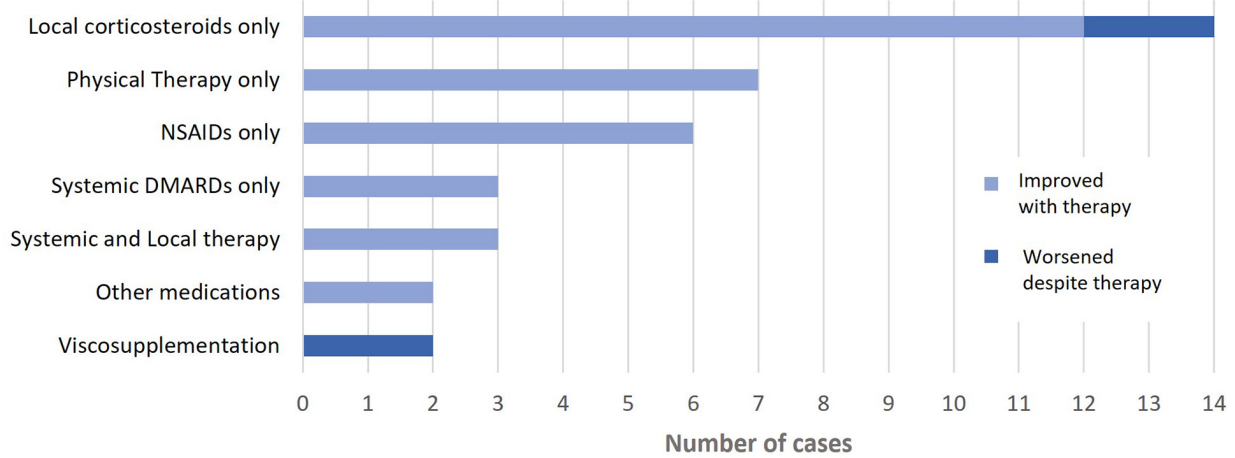

Figure 1 Timing and therapies for post-ICl-aOA. (A) Incidence of $\mathrm{ICl}-\mathrm{aOA}(\mathrm{aOA}$ after $\mathrm{ICl}$ ) ranged from the first month after $\mathrm{ICl}$ initiation up to month 22 after ICl initiation, with most cases occurring in the first 6 months after start of ICl. Five of 36 patients experienced ICl-aOA after $\mathrm{ICl}$ cessation (0.6, 3.5, 4.4, 7.3 and 15.4 months after $\mathrm{ICl}$ cessation), corresponding to presentation after ICl initiation as follows: 2.0, 9.6, 19.1, 8.7, and 16.1 months after ICI initiation, respectively (as denoted in darker color). (B) The therapeutic option most used was local or intra-articular corticosteroid therapy followed by conservative management with physical therapy only, then NSAIDs. Most patients experienced improvement in signs and symptoms with treatment. $\mathrm{aOA}$, activated osteoarthritis; DMARD, disease-modifying antirheumatic drug; ICl, immune checkpoint inhibitor; ICl-aOA, IClactivated osteoarthritis; NSAID, non-steroidal anti-inflammatory drug.

other irAEs were rheumatic (7/20) and dermatological irAEs $(7 / 20)$. These other rheumatic irAEs included two patients with sicca syndrome, two patients with polymyalgia rheumatica, one patient with inflammatory arthropathy, one patient with Raynaud's phenomenon and one with chilblains. Of the seven that also experienced dermatological irAEs, three had non-specific rash or dermatitis; one had pruritus; two had psoriasis flares; and one urticarial vasculitis. The three patients with high-grade ICI-aOA all had other irAEs, but there was no statistically significant association with severity of ICI-aOA and presence of other irAEs. In those cases with concomitant inflammatory arthritis, we diagnosed aOA as a separate phenomenon based on our defined criteria as mentioned previously.

\section{ICI-a0A therapeutics}

Management approach to ICI-aOA ranged from local corticosteroid injections (CSIs) to systemic diseasemodifying antirheumatic drugs (figure 1B). Four patients (2 of 12 with local CSIs, 2 of 2 patients with viscosupplementation) had worsening of their ICI-aOA despite respective therapy. All four of these patients also experienced co-occurrence of another irAE. Additionally, all of the patients without another irAE experienced improvement or had stability of their aOA in response to their respective therapy. When compared with patients without other irAEs, patients with an irAE in addition to their aOA were more than four times more likely to have lack of improvement or worsening of their aOA (Likelihood ratio 4.67 , $\mathrm{p}$ value $=0.031$ ). All three patients who experienced high-grade ICI-aOA were given local and systemic corticosteroids at doses of prednisone starting at $20 \mathrm{mg}$ or less; all appreciated improvement in symptoms.

\section{DISCUSSION}

In this paper we describe a cohort of patients who experienced symptomatic OA after ICI start, called ICI-aOA. In our cohort, majority of the patients with this $\mathrm{AE}$ were treated with a PD-1/PD-L1 inhibitor monotherapy. Most of the patients had more than one joint involvement, often including a large joint. Median time to onset of symptoms was 5 months, 


\section{Representative Images of Osteoarthritis from ICl-aOA Cohort}

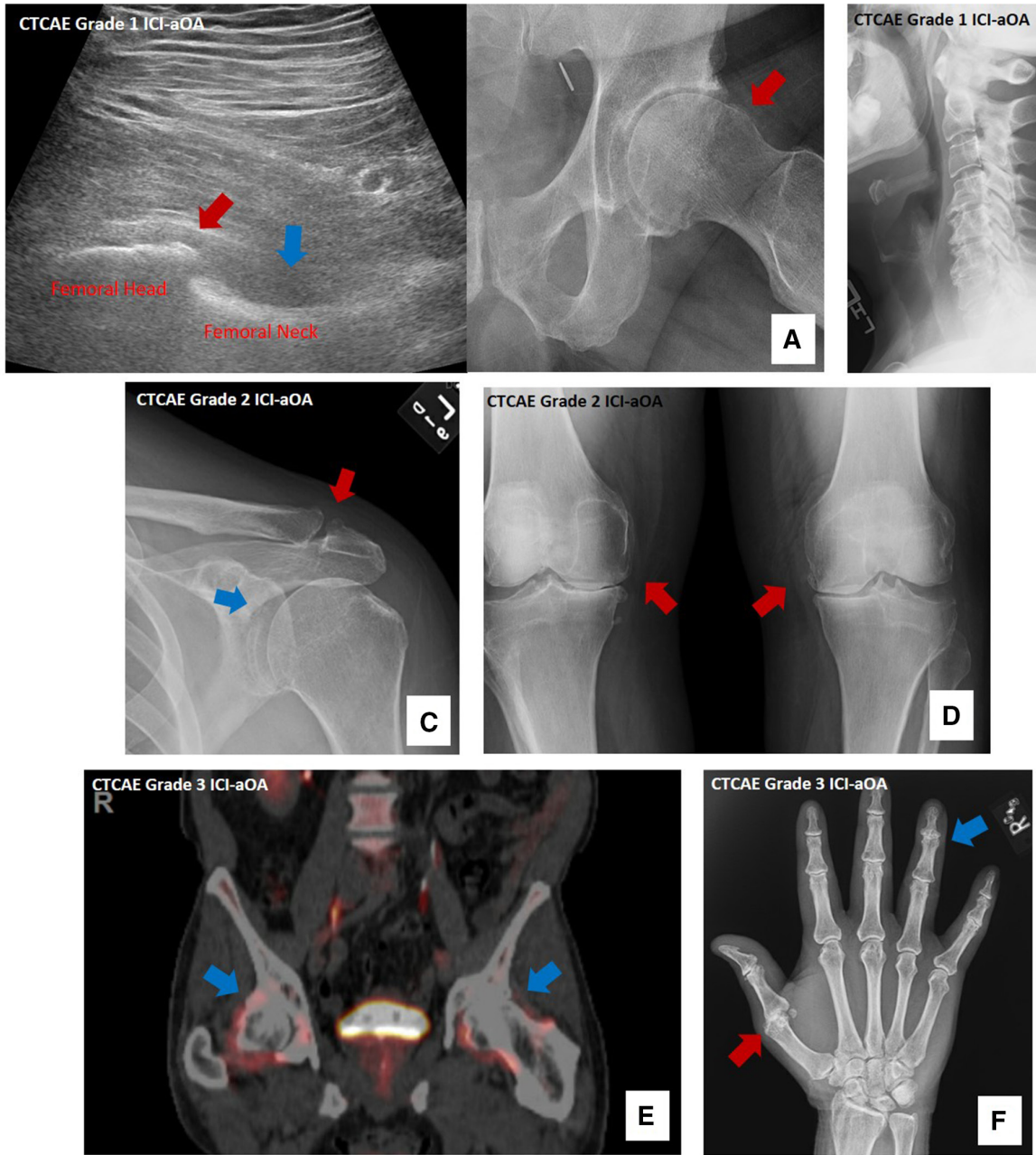

Figure 2 Representative images of OA from ICl-aOA. (A) Ultrasound and corresponding radiograph of left hip demonstrating $\mathrm{OA}$ with osteophyte (red arrow on ultrasound and X-ray) and small joint effusion (blue arrow). (B) Lateral X-ray of the cervical spine depicting osteophytes and lost of disk space, indicating cervical spine OA. (C) Left shoulder X-ray noting mild OA affecting the glenohumeral joint (blue arrow) and acromioclavicular joint (red arrow). (D) Standing X-ray of the knees depicting moderate OA of bilateral knee joints (red arrows). (E) Positron emissions tomography-computerized tomography showing mild fluorodeoxyglucose uptake (blue arrows) associated with degenerative changes and OA at the hips. (F) Right-hand X-ray demonstrating moderate $\mathrm{OA}$ in various interphalangeal joints, most notable of which is the fourth distal interphalangeal joint (blue arrow) with severe OA in the first metacarpal joint (red arrow). CTCAE, Common Terminology Criteria for Adverse Events; $\mathrm{ICl}-\mathrm{aOA}$, ICl-activated osteoarthritis; OA, osteoarthritis.

with five patients who experienced ICI-aOA after ICI cessation. More than half of our patients also experienced an irAE at some point after ICI initiation. For the majority of the patients, local therapy was sufficient and successful, while the patients who failed their aOA therapy were all notably patients who had other adverse events. Patients with highgrade ICI-aOA all had other irAEs, were more likely to have some serological positivity, and were managed with systemic corticosteroids. ICI-aOA should be recognized as an ICI toxicity necessitating timely referral to and assessment by rheumatology for better characterization of the arthropathy and effective management thereafter.

Due to the heterogeneity of musculoskeletal irAE, earlier referral to rheumatology can appreciably accelerate evaluation and delineate between ICI-aOA and immune-related inflammatory arthritis. With local therapy for ICI-aOA being encouragingly successful, there is potential to manage patients' joint symptoms without the hypothetical risk of mitigating checkpoint inhibitor efficacy by way of systemic immunosuppression. ${ }^{15} 16$ Furthermore, autoantibody positivity, such as ANA, RF or CCP, can be misleading. Our study found multiple cases of ICI-aOA with low-titer seropositivity but lack of inflammatory signs or symptoms that would indicate inflammatory arthritis. In those cases with concomitant inflammatory irAE, we diagnosed aOA as a separate phenomenon based on our defined criteria. This further demonstrates the importance of a dedicated and thorough rheumatological evaluation to contextualize laboratory values with that of symptomology and physical examination of autoimmune disease. Finally, the co-occurrence of ICI-aOA with irAEs highlights the importance of a rheumatologist's role in ICI toxicity management and that 
of a multidisciplinary model when caring for patients with concern for or diagnosis of irAEs.

OA has traditionally been considered a non-inflammatory condition; however, with the advent of more advanced imaging techniques and molecular and genetic lab technologies, it has become evident that chronic, low-grade inflammation does play a role in the pathogenesis of OA. ${ }^{11-14}$ Histologically, OA synovium demonstrates infiltration by mononuclear cells, including activated $\mathrm{B}$ and $\mathrm{T}$ cells, and evidence of angiogenesis. ${ }^{17}{ }^{18}$ In addition, there appears to be different phenotypes of OA, including a more inflammatory phenotype, with a much higher expression of inflammatory cytokines such as interleukin (IL)-1B, IL-6, and IL-8. ${ }^{19}$ Intriguingly, Liu et al demonstrated in a mouse model of OA that PD-L1 blockade induced more severe arthritis and increased inflammatory cytokine production. ${ }^{14}$ The few patients in our study with high-grade ICI-aOA had autoimmune serological positivity and required systemic corticosteroids for therapy. However, even in these patients, doses of prednisone $\leq 20 \mathrm{mg}$ were sufficient for successful therapy, reflecting its differing pathophysiology from that of immunerelated inflammatory arthritis and other irAEs, which tend to require higher doses and longer courses of systemic immunosuppression for treatment. ${ }^{5021}$ While too few in number to draw any definitive conclusions, this could shed further light on the role and extent of inflammation that have been implicated in past murine models and phenotypical studies of OA.

To further demonstrate the unique clinical features of ICI-aOA and to distinguish it as a separate entity from ICIIA, we provide a framework for comparison between the two groups (table 2). Many reports have described clinical features of ICI-IA, ${ }^{722-24}$ but to date, the most comprehensive review of ICI-IA is a systematic literature review of case reports and case series by Ghosh $e t a l^{5}$ Table 2 summarizes key similarities and differences between ICI-IA and ICI-aOA.

The populations are similar in age, gender, type of malignancy, and timing of presentation. Notable differences between the two groups arise from joint distribution as well as therapeutic approach for the arthropathies. While ICI-IA tends to impact predominantly, the small metacarpophalangeal and PIP joints of the hands, ICI-aOA tends to impact large joints such as the hips and knees. Over half of the patients in Ghosh $e t a l$ s review with ICI-IA did not receive any pharmacological therapy, whereas over $40 \%$ of patients with ICI-aOA in our cohort received local CSIs and only onefifth of our patients had no pharmacological intervention. Regardless of chosen treatment, the majority of patients improve with therapy (63\% in ICI-IA review and $89 \%$ in our ICI-aOA cohort).

\section{Limitations}

Our study is not without limitations. We examine a small number of cases, limiting our ability to make definite conclusions. Additionally, given the retrospective and observational nature of our study, there is potential for confounders and not all pertinent information is available for analysis as it was not drawn prospectively. We also do not
Table $2 \mathrm{ICl}-\mathrm{aOA}$ versus $\mathrm{ICl}-\mathrm{IA}$

\begin{tabular}{lll}
\hline & ICI-aOA & ICI-IA (Ghosh et $\mathbf{a l}^{5}$ ) \\
\hline Demographics & & \\
Mean age (years) & $66(51-81)$ & $63(52-74)$ \\
Sex & $53 \%$ male & $61 \%$ male \\
Tumor type & Melanoma (28\%) & Melanoma (57\%) \\
& NSCLC (14\%) & NSCLC (22\%) \\
& RCC (17\%) & RCC (7\%) \\
Cancer & PD-1/PD-L1i & PD-1/PD-L1i \\
immunotherapy & monotherapy (86\%) & monotherapy (78\%) \\
& CTLA-4i monotherapy & CTLA-4i monotherapy \\
& $(0 \%)$ & $(5 \%)$ \\
Malignancy & Complete response & Complete response \\
outcome & $(44 \%)$ & $(20 \%)$ \\
& Partial response (14\%) & Partial response (33\%) \\
& Stable disease (13\%) & Stable disease (21\%) \\
& Progressive disease & Progressive disease \\
& $(11 \%)$ & $(27 \%)$
\end{tabular}

Median months to 5 months (range 0-34) 4 months (range 0-53) onset

\begin{tabular}{|c|c|c|}
\hline Joint distribution & $\begin{array}{l}\text { Small joint (first CMC, } \\
\text { DIPs, PIPs, etc) }(25 \%) \\
\text { Large joint }(53 \%) \\
\text { Spine }(14 \%)\end{array}$ & $\begin{array}{l}\text { Small joint (RA } \\
\text { distribution: MCPs, } \\
\text { PIPs, etc) }(65 \%) \\
\text { Large joint }(22 \%) \\
\text { Spine (13\%) }\end{array}$ \\
\hline Joint count & Polyarticular (67\%) & Polyarticular (49\%) \\
\hline $\begin{array}{l}\text { Serologies (\% of } \\
\text { total tested) }\end{array}$ & $\begin{array}{l}19 \%(5 / 27) \mathrm{RF} \text { and/or } \\
\text { CCP } \\
32 \%(7 / 22) \text { ANA }\end{array}$ & $\begin{array}{l}9 \%(25 / 270) \mathrm{RF} \text { and/ } \\
\text { or CCP } \\
30 \%(57 / 193) \text { ANA }\end{array}$ \\
\hline Therapeut & $\begin{array}{l}\text { No medicinal therapy } \\
(20 \%) \\
\text { NSAIDs }(20 \%) \\
\text { Local CSIs (43\%) } \\
\text { Systemic } \\
\text { corticosteroids (11\%) } \\
\text { Non-steroidal DMARDs } \\
(3 \%)\end{array}$ & $\begin{array}{l}\text { No medicinal therapy } \\
(55 \%) \\
\text { NSAIDs }(20 \%) \\
\text { Local CSIs (14\%) } \\
\text { Systemic } \\
\text { corticosteroids (74\%) } \\
\text { Non-steroidal DMARDs } \\
(31 \%)\end{array}$ \\
\hline $\begin{array}{l}\text { Other irAEs, any } \\
\text { grade }\end{array}$ & $58 \%$ & $52 \%$ \\
\hline $\begin{array}{l}\text { Arthropathy } \\
\text { outcome }\end{array}$ & $\begin{array}{l}\text { Improved or controlled } \\
\text { with therapy } 89 \%\end{array}$ & $\begin{array}{l}\text { Improved or controlled } \\
\text { with therapy } 63 \%\end{array}$ \\
\hline
\end{tabular}

ANA, antinuclear antibody; aOA, activated osteoarthritis; CCP, cyclic citrullinated peptide; CMC, carpometacarpal; CSI, corticosteroid injection; CTLA-4i, cytotoxic T lymphocyte-associated protein 4 inhibitor; DIP, distal interphalangeal; DMARD, disease modifying antirheumatic drug; ICI, immune checkpoint inhibitor; ICl-aOA, $\mathrm{ICl}$-activated osteoarthritis; ICI-IA, ICl-inflammatory arthritis; irAE, immune-related adverse event; MCP, metacarpophalangeal; NSAID, non-steroidal anti-inflammatory drug; NSCLC, non-small cell lung carcinoma; PD-1/PD-L1i, programed cell death protein 1 inhibitor; PIP, proximal interphalangeal; RA, rheumatoid arthritis; RCC, renal cell carcinoma; RF, rheumatoid factor.

have a comparator cohort of patients who had ICI therapy without OA enhancement nor a group with history of malignancy and without cancer immunotherapy. All of these open up room for bias. For example, the high number of co-occurring rheumatic irAEs in our sample, contrary to their rarity in past research, is likely a result of referral bias. However, the correlations between multisystemic irAEs and ICI-aOA, as well as the potential pathobiological insight to primary OA our findings could indicate, are notable and 
enough to warrant a more robust study of these associations in the future.

\section{CONCLUSION}

We present the first description of OA exacerbation after cancer immunotherapy. Our findings suggest the need for a heightened clinical vigilance of aOA after ICI therapy and justify prospective, multi-institutional examination of ICI-aOA.

\section{Twitter Pankti Reid @ReidMDMPH and David FL Liew @drdavidliew}

Contributors Conception or design of the work and final approval of the version to be published: PR, DL, RA, ARB, and KKC; data collection: PR, DL, and KKC; data analysis and interpretation andcritical revision of the article: PR, DL, ARB, and KKC; drafting of the article: PR and KKC.

Funding The authors have not declared a specific grant for this research from any funding agency in the public, commercial or not-for-profit sectors.

Competing interests PR is a coinventor of a filed patent covering the use of lowdose tocilizumab in viral infections.

\section{Patient consent for publication Not required.}

Ethics approval Collection of patient data was approved by local institutional review boards at respective institutions: Hospital for Special Surgery in New York, University of Chicago in Chicago, Illinois, and Austin Health in Melbourne, Victoria, Australia.

\section{Provenance and peer review Not commissioned; externally peer reviewed.}

Data availability statement Data are available upon reasonable request. All data relevant to the study are included in the article or uploaded as supplementary information. All data relevant to the study are included in the article or uploaded as supplementary information. Details of statistical analyses and other data are available upon reasonable request.

Supplemental material This content has been supplied by the author(s). It has not been vetted by BMJ Publishing Group Limited (BMJ) and may not have been peer-reviewed. Any opinions or recommendations discussed are solely those of the author(s) and are not endorsed by BMJ. BMJ disclaims all liability and responsibility arising from any reliance placed on the content. Where the content includes any translated material, BMJ does not warrant the accuracy and reliability of the translations (including but not limited to local regulations, clinical guidelines, terminology, drug names and drug dosages), and is not responsible for any error and/or omissions arising from translation and adaptation or otherwise.

Open access This is an open access article distributed in accordance with the Creative Commons Attribution Non Commercial (CC BY-NC 4.0) license, which permits others to distribute, remix, adapt, build upon this work non-commercially, and license their derivative works on different terms, provided the original work is properly cited, appropriate credit is given, any changes made indicated, and the use is non-commercial. See http://creativecommons.org/licenses/by-nc/4.0/.

\section{ORCID iDs}

Pankti Reid http://orcid.org/0000-0002-7645-0919

David FL Liew http://orcid.org/0000-0001-8451-8883

\section{REFERENCES}

1 Martins F, Sofiya L, Sykiotis GP, et al. Adverse effects of immunecheckpoint inhibitors: epidemiology, management and surveillance. Nat Rev Clin Oncol 2019;16:563-80.

2 Cappelli LC, Gutierrez AK, Bingham CO, et al. Rheumatic and musculoskeletal immune-related adverse events due to immune checkpoint inhibitors: a systematic review of the literature. Arthritis Care Res 2017;69:1751-63.

3 Braaten TJ, Brahmer JR, Forde PM, et al. Immune checkpoint inhibitor-induced inflammatory arthritis persists after immunotherapy cessation. Ann Rheum Dis 2020;79:332-338.

4 Collison J. Checkpoint inhibitor-induced arthritis is persistent. Nat Rev Rheumatol 2019;15:698.

5 Ghosh N, Tiongson MD, Stewart C, et al. Checkpoint inhibitor-associated arthritis: a systematic review of case reports and case series. J Clin Rheumatol 2020. doi:10.1097/ RHU.0000000000001370. [Epub ahead of print: 25 Apr 2020].

6 Buder-Bakhaya K, Benesova K, Schulz C, et al. Characterization of arthralgia induced by PD-1 antibody treatment in patients with metastasized cutaneous malignancies. Cancer Immunol Immunother 2018;67:175-82.

7 Cappelli LC, Bingham CO. Expert perspective: immune checkpoint inhibitors and rheumatologic complications. Arthritis Rheumatol 2021;73:553-65.

8 Arnaud L, Lebrun-Vignes B, Salem J-E. Checkpoint inhibitorassociated immune arthritis. Ann Rheum Dis 2019;78:e68.

9 Cappelli LC, Thomas MA, Bingham CO, et al. Immune checkpoint inhibitor-induced inflammatory arthritis as a model of autoimmune arthritis. Immunol Rev 2020;294:106-23.

10 Larsen GL, Henson PM. Mediators of inflammation. Annu Rev Immunol 1983;1:335-59.

11 Deveza LA, Nelson AE, Loeser RF. Phenotypes of osteoarthritis: current state and future implications. Clin Exp Rheumatol 2019;37 Suppl 120:64-72.

12 Berenbaum F. Deep phenotyping of osteoarthritis: a step forward. Ann Rheum Dis 2019;78:3-5.

13 Van Spil WE, Kubassova O, Boesen M, et al. Osteoarthritis phenotypes and novel therapeutic targets. Biochem Pharmacol 2019;165:41-8.

14 Liu S, Mi J, Liu W, et al. Blocking of checkpoint receptor PD-L1 aggravates osteoarthritis in macrophage-dependent manner in the mice model. Int $\mathrm{J}$ Immunopathol Pharmacol 2019;33:2058738418820760.

15 Ricciuti B, Dahlberg SE, Adeni A, et al. Immune checkpoint inhibitor outcomes for patients with Non-Small-Cell lung cancer receiving baseline corticosteroids for palliative versus Nonpalliative indications. JCO 2019;37:1927-34.

16 Fucà G, Galli G, Poggi M, et al. Modulation of peripheral blood immune cells by early use of steroids and its association with clinical outcomes in patients with metastatic non-small cell lung cancer treated with immune checkpoint inhibitors. ESMO Open 2019;4:e000457.

17 Liu-Bryan R, Terkeltaub R. Emerging regulators of the inflammatory process in osteoarthritis. Nat Rev Rheumatol 2015;11:35-44.

18 Mathiessen A, Conaghan PG. Synovitis in osteoarthritis: current understanding with therapeutic implications. Arthritis Res Ther 2017;19:18.

19 Dell'Isola A, Allan R, Smith SL, et al. Identification of clinical phenotypes in knee osteoarthritis: a systematic review of the literature. BMC Musculoskelet Disord 2016;17:425.

20 Puzanov I, Diab A, Abdallah K, et al. Managing toxicities associated with immune checkpoint inhibitors: consensus recommendations from the Society for immunotherapy of cancer (SITC) toxicity management Working group. J Immunother Cancer 2017;5:95.

21 Brahmer JR, Lacchetti C, Schneider BJ, et al. Management of immune-related adverse events in patients treated with immune checkpoint inhibitor therapy: American Society of clinical oncology clinical practice guideline. JCO 2018;36:1714-68.

22 Pundole X, Abdel-Wahab N, Suarez-Almazor ME. Arthritis risk with immune checkpoint inhibitor therapy for cancer. Curr Opin Rheumatol 2019;31:293-9.

23 Belkhir R, Burel SL, Dunogeant L, et al. Rheumatoid arthritis and polymyalgia rheumatica occurring after immune checkpoint inhibitor treatment. Ann Rheum Dis 2017;76:1747-50.

24 Calabrese L, Mariette X. Chronic inflammatory arthritis following checkpoint inhibitor therapy for cancer: game changing implications. Ann Rheum Dis 2020;79:309-11. 